\title{
The case for stool banks in South Africa
}

The ancient (AD 300) practice of faecal microbiota transplant (FMT) corrects dysbiosis (quantitative, qualitative, metabolic or locational imbalance of gastrointestinal commensals) by repopulation with normal intestinal microbiota. Clostridium difficile infection (CDI) is a serious and increasingly prevalent healthcare-associated disease. ${ }^{[1]}$ The efficacy of FMT for recurrent CDI is crucial proof of concept that therapeutic modification of the gastrointestinal microbiome can be an effective treatment modality for human disease. ${ }^{[2]}$

In the past, metronidazole and vancomycin were the mainstay of CDI treatment, but outcomes with metronidazole are suboptimal and it has been superseded by more effective but expensive agents such as fidaxomicin. ${ }^{[2]}$ Other novel agents such as bezlotoxumab, ${ }^{[3]}$ a monoclonal antibody for the prevention of recurrent CDI, are also mostly unaffordable for resource-constrained healthcare sectors in South Africa (SA). FMT efficacy has been confirmed in recurrent CDI and has the added advantage that it is relatively inexpensive. ${ }^{[4]}$

FMT involves the introduction of microbiota from a healthy donor to a patient by colonoscopy, enema or nasogastric tube ${ }^{[5]}$ Currently, $\mathrm{CDI}$ is the main indication for FMT, but as a novel treatment strategy, it has also been investigated in conditions ranging from inflammatory bowel disease to metabolic diseases, obesity, malnutrition and autism spectrum disorders. ${ }^{[6]}$ To this end, the SA Gastroenterology Society (SAGES) published FMT guidelines in 2015. ${ }^{[7]}$

The procedure is being performed in the private and public sectors in SA. As there is no established national gastrointestinal microbiome bank, such as OpenBiome, ${ }^{[8]}$ a non-profit stool bank in the USA with the aim of expanding safe access to faecal transplants, it appears that currently healthcare providers mostly use stool from close family and friends and rarely from unrelated donors. Although experiences with FMT in SA are mostly unknown, a recent study from Johannesburg demonstrated that at least $70 \%$ of donors were close relatives. ${ }^{[9]}$ The article highlights the multiple challenges we face, including the pertinent discussion on what constitutes an ideal donor and the minimal screening tests required for all stool donors in SA. To date, there have been no trials evaluating outcomes of faecal transplant in relation to donor characteristics. ${ }^{[10]}$

Therefore, current criteria for selection and screening of faecal donors are primarily a product of expert opinion. While detailed protocols that describe FMT administration and consensus reports on overall good conduct exist abroad, regulatory challenges and uncertainty still prevail - not only in SA, but also abroad. Perhaps one reason for lack of a legal framework relates to the urgent need for complete methodological reporting for clinical trials and interventions involving FMT. A recent systematic review (conducted rigorously under PRISMA (Preferred Reporting Items for Systematic reviews and Meta-Analyses) guidelines) of methods and studies assessing FMT, highlighted that key components of FMT interventions, which are necessary to replicate and understand study findings regarding efficacy and safety, are poorly reported. ${ }^{[11]}$

In addition to the abovementioned regulatory issues, it is being debated whether FMT should be regulated as a drug or a tissue. However, neither of these established regulatory schemas applies clearly to the use of faeces as a therapy. The standard regulatory pathways governing traditional drugs are therefore inappropriate. Obtaining approval via the drug regulatory pathway will create a myriad of problems unsolvable in the medium term. The SA Health Products Regulatory Authority (SAHPRA) proposes that a drug should be: (i) identical in active ingredient; (ii) consistent with regard to the concentration, quality and performance characteristics; and (iii) universal in dosage and route of administration. Given the extraordinary diversity of the microbiome, conventional labelling does not apply.

We therefore propose a multidisciplinary intersectorial biobank working group (WG) for SA, comprising experts in the fields of clinical microbiology, gastroenterology, infectious diseases and bioinformatics, who could dictate policy and implementation. Safe and reproducible methods for donor screening, laboratory processing, funding and clinical application of FMT are warranted locally, and probably necessitate establishment of a consensus clinical framework for a stool bank. The framework could be divided into main-activity sections such as donor recruitment (i.e. screening, exclusion criteria), laboratory processing of donor material (e.g. initial handling, cryopreservation and thawing for clinical application), clinical application (i.e. routes, safety) and donor-recipient data management. Stool banks should only provide access to screened stool that can be traced to the donor and for documenting adverse events in the short, medium and long term. ${ }^{[12]}$ This division provides a practical organisational structure and clarifies both the tasks and the necessary personnel to maintain an operational stool bank nationally or regionally.

Another advantage of an SA WG relates to several studies of FMT that have performed molecular analysis of the microbiota; therefore, centralised biorepositories or data repositories could be established to enable collective assessment of the effect of FMT on microbiota. This could facilitate an understanding of the rationale for this treatment and lead to refinement of microbiome-based therapies. Globally, systematic analysis of gastrointestinal metagenomics in defined hospitalised patients and understanding of the potential protective, metabolic and immune functions and hazards associated with its manipulation via FMT, are required to support the current framework established by regulatory agencies.

The authors believe that systematic appraisal by the proposed WG of the evolving body of scientific FMT research is needed to inform development of stool banks and guide evidence-based clinical best practices in SA. We propose this to constitute the only manner to facilitate introduction of potentially multiple cost-effective novel management and infection control interventions in resourceconstrained settings.

\section{Ernst Fredericks}

Department of Biochemistry and Microbiology, Faculty of Science, Nelson Mandela University, Port Elizabeth, South Africa

esfred@eject.co.za

\section{Ebrahim Hoosien}

Clinical Microbiology, Ampath National Reference Laboratory, Pretoria, South Africa

\section{Adrian Brink}

Division of Medical Microbiology, Department of Pathology and National Health Laboratory Service, Faculty of Health Sciences, University of Cape Town, South Africa

1. Lessa FC, Winston LG, McDonald LC. Burden of Clostridium difficile infection in the United States. N Engl J Med 2015;372(24):2369-2370. https://doi.org/10.1056/NEJMc1505190

2. Rajasingham R, Enns EA, Khoruts A, Vaughn BP. Cost-effectiveness of treatment regimens for Clostridioides difficile infection - an evaluation of the 2018 Infectious Diseases Society of America guidelines. Clin Infect Dis 2019;pii:ciz31. https://doi.org/10.1093/cid/ciz318 
3. Ooijevaar RE, van Beurden YH, Terveer EM, et al. Update of treatment algorithms for Clostridium difficile infection. Clin Microbiol Infect 2018;24(5):452. https://doi.org/10.1016/j.cmi.2017.12.022

4. Cammarota G, Masucci L, Ianiro G, et al. Randomised clinical trial: Faecal microbiota transplantation by colonoscopy vs. vancomycin for the treatment of recurrent Clostridium difficile infection. Aliment Pharm Ther 2015:41(9):835-843. https://doi.org/10.1111/apt13144

5. Goldenberg SD, Batra R, Beales I, et al. Comparison of different strategies for providing fecal microbiota transplantation to treat patients with recurrent Clostridium difficile infection in two Englis hospitals: A review. Infect Dis Ther 2018;7(1):71-86. https://doi.org/10.1007/s40121-018-0189-y

6. Millan B, Laffin M, Madsen K. Fecal microbiota transplantation: Beyond Clostridium difficile. Curr Infect Dis Rep 2017;19(9):31. https://doi.org/10.1007/s11908-017-0586-5

7. Fredericks E. SAGES clinical guidelines for faecal microbiota transplantation. S Afr Gastroenterol Rev 2015;13(3):27-28

8. OpenBiome. https://www.openbiome.org/home (accessed 14 May 2019).

9. Lee S, Drennan K, Simons G, et al. The 'ins and outs' of faecal microbiota transplant for recurrent Clostridium difficile diarrhoea at Wits Donald Gordon Medical Centre, Johannesburg, South Africa. S Afr Med J 2018;108(5):403-407. https://doi.org/10.7196/SAMJ.2018.v108i5.12367
10. Woodworth MH, Neish EM, Miller NS, et al. Laboratory testing of donors and stool samples for fecal microbiota transplantation for recurrent Clostridium difficile infection. J Clin Microbiol 2017;55(4):1002-1010. https://doi.org/10.1128/JCM.02327-16

11. Bafeta A, Yavchitz A, Riveros C, Batista R, Ravaud P. Methods and reporting studies assessing fecal microbiota transplantation. A systematic review. Ann Intern Med 2017;167(1):34-39. https://doi. org $/ 10.7326 / \mathrm{M} 16-2810$

12. Terveer EM, van Beurden YH, Goorhuis A, et al. How to: Establish and run a stool bank. Clin Microbiol Infect 2017;23(12):924-930. https://doi.org/10.1016/j.cmi.2017.05.015

S Afr Med J 2019;109(8):546-547. DOI:10.7196/SAMJ.2019.v109i8.14169 